UDC 821.131.1.09-13 Alighieri D.

DOI 10.18485/italbg.2015.2.1

\author{
Enrico Malato*
}

Centro "Pio Rajna"

\title{
LEGGERE DANTE NEL XXI SECOLO: BILANCI E PROSPETTIVE DEGLI STUDI DANTESCHI**
}

Abstract: Nel mentre si avvicina il settimo Centenario della morte di Dante (nel 2021), e in corso del settimo Centenario della Divina Commedia, l'autore si propone di fare qualche riflessione in ottica retrospettiva, in chiave di bilancio degli studi danteschi negli ultimi decenni, ma anche in ottica di prospettiva, di previsione e di programmazione delle vie che la critica dantesca potrà percorrere.

Parole chiave: Dante, settimo Centenario, Divina Commedia, critica dantesca, prospettive, programmazione

Avvicinandosi il settimo Centenario della morte di Dante, nel 2021 - e si sa quanto per Dante sette fosse un numero sacro per eccellenza, dunque una cadenza particolarmente carica di valori simbolici e significativi -, mentre è in corso il settimo Centenario della Divina Commedia, il "poema sacro" di cui egli dovette iniziare la stesura proprio intorno agli anni 1306 o '07 o '08, pubblicando 1'Inferno e il Purgatorio intorno al 1313-14, sembrano maturi $i$ tempi per qualche riflessione: in ottica retrospettiva, in chiave di bilancio degli studi danteschi negli ultimi decenni (o diciamo pure negli ultimi due o tre secoli), e però anche in ottica di prospettiva, di previsione e programmazione dei sentieri che la critica dantesca potrà percorrere, o almeno vede schiusi davanti a sé, alle soglie del nuovo millennio. E inevitabilmente ne verrà insieme qualche considerazione su un altro aspetto - sconcertante $\mathrm{e}$ affascinante insieme - della dantologia moderna: sul perché si continui a leggere Dante, oggi, con immutato interesse, magari con crescente entusiasmo e partecipazione delle masse, che lo hanno promosso addirittura a

*enrico.malato@unina.it

** Il contributo riprende gli spunti della relazione pronunciata al convegno di studi Le riflessioni italo-serbe. L'eredità di Nikša Stipčević tenutosi all'Accademia Serba delle Scienze e delle Arti, a Belgrado, il 3 novembre 2014. 
protagonista di romanzi popolari a circolazione internazionale, e sul come lo si legga, con quale effettiva aderenza al messaggio poetico che egli ci ha lasciato; sulle ragioni di questa intramontabile fidelitas di un vasto pubblico a un poeta che è oggettivamente "difficile", tale da chiedere una speciale attrezzatura mentale e un bagaglio culturale non comuni, e in un momento di crisi - almeno in Italia - degli studi letterari e in particolare della critica e soprattutto della filologia dantesca nella scuola e nell'università.

Grandi critici e artisti del Novecento, italiani e stranieri - da Michele Barbi a Erich Auerbach, da Ernst Robert Curtius a Gianfranco Contini, ad Antonino Pagliaro, al poeta Eugenio Montale, ecc. -, hanno parlato, con riferimento a Dante, di "miracolo" o di "prodigio" dantesco: tale non soltanto per la densità e l'altezza della poesia di Dante, senza uguali nella storia della poesia universale, non soltanto perché la Commedia è l'unico capolavoro del medioevo in lingua accessibile, scritto nell'unica lingua di cultura dell'Occidente che, grazie proprio a quell'opera, è rimasta sostanzialmente inalterata per oltre settecento anni, così che è oggi coincidente (a differenza dal francese, dall'inglese, dallo spagnolo, dal tedesco, ecc.) con la lingua italiana parlata, ma anche per la sua "popolarità" intramontabile, appena ricordata, per la sua capacità di esercitare un fascino straordinario su legioni di lettori, in tutte le latitudini del globo, che non conosce crisi ${ }^{1}$. Dante contende a Shakespeare, spesso con successo, il "primato" nella "grandezza poetica", quale viene dichiarato nelle valutazioni soggettive di quei lettori, e anche nella frequentazione delle loro opere: ciò che appare tanto più sorprendente, in quanto non solo Dante ha scritto in una lingua molto meno diffusa di quella di Shakespeare (letto perciò spesso, fuori d'Italia, in traduzione), ma perché mentre il drammaturgo è un poeta moderno, vicino alla sensibilità dell'uomo del nostro tempo, Dante è un uomo tipicamente del Medioevo, portatore di una tematica e una problematica in apparenza propri di un'età che sembra lontanissima dalla nostra. E pure riesce a proporli, quei temi e quei problemi, in modi, in forme, con un linguaggio che esercitano una forte "presa" su lettori di ogni tempo e di ogni paese, un fascino, una suggestione che coinvolge lettori comuni e critici letterari, come attestano pochi dati certi: La Divina Commedia è l'opera in lingua straniera più tradotta e ristampata in inglese dopo la Bibbia; su Dante si pubblicano ogni anno nel mondo, solo in sede scientifica, circa 1000/1500 contributi critici (libri, saggi, articoli, recensioni, traduzioni, ecc.), esclusi i giornali e le pubblicazioni divulgative e scolastiche, dei quali un terzo fuori d'Italia, e di questi, la metà tra USA e Canada².

\footnotetext{
${ }^{1} \mathrm{Su}$ questo quadro v. Malato $\left(2006^{2}, 2005^{1}\right.$ : 658-92), partic. pp. $658 \mathrm{sgg}$.

${ }^{2}$ Cfr. la Bibliografia Generale della Lingua e della Letteratura Italiana (1991-).
} 
Conviene dunque fermare brevemente l'attenzione, ai fini del nostro discorso, da un lato sulla produzione critica dantesca, sulla quantità (e la qualità) della "letteratura" relativa a Dante che, sempre più abbondante, si va producendo nel mondo, con contributi talvolta importanti al progresso della conoscenza della figura e dell'opera di Dante, non di rado con effetto di "ingorgo", creando difficoltà agli studiosi nell'orientarsi in questa selva selvaggia, ardua da districare; dall'altro - in conseguenza - sulla fruizione che di essa si è fatta, sui risultati effettivamente e stabilmente conseguiti e quelli tuttora incerti e discutibili e discussi, sulle prospettive nuove che si sono aperte alla ricerca, sui campi in cui essa potrà utilmente svilupparsi.

È appena il caso di ricordare che, dopo la parentesi seicentesca, la rinascita, fin dagli inizi del XVIII secolo, dell'interesse per Dante - che approdò nel giro di pochi anni alle nuove edizioni commentate (tutte poi variamente ristampate) della Commedia, a cura di Giov. Antonio Volpi (1726-27), di Pompeo Venturi (1732), fino a quella curata da Baldassarre Lombardi (1791) - trovò centri particolarmente attivi di ricerca di documenti danteschi e di studio dell'opera di Dante a Venezia, a Roma, a Firenze, soprattutto a Verona, dove per impulso di Scipione Maffei si costituisce un nucleo di valenti studiosi - Bartolomeo Perazzini, Lodovico Salvi, Giuseppe Torelli, il canonico Gio. Jacopo Dionisi - che afferma la necessità di uno studio sistematico dell'opera dantesca, sostenuta da un'apposita istituzione, per la quale si ipotizza la forma di una "Accademia dantesca"".

È l'inizio di una "riscoperta" di Dante che nel volgere di pochi decenni si traduce in fervore di studi, di iniziative, di manifestazioni celebrative, in attività di ricerca di antichi documenti danteschi, di antichi codici testimoni delle sue opere, che coinvolge non solo ammiratori italiani - di un'Italia ancora divisa in tanti Stati e staterelli -, ma anche stranieri. La fama di Dante si spande rapidamente in tutta Europa: in Inghilterra, dove già nel Seicento John Milton era stato grande estimatore del poeta della Commedia, Coleridge, Shelley, Byron sono impegnati fin dagli albori dell'Ottocento in una entusiastica divulgazione della sua opera, cui contribuiscono anche Foscolo e altri esuli italiani a Londra; in Svizzera e in Francia ammiratori di Dante, contro la contestazione di Voltaire, sono M.me de Staël e Sismonde de Sismondi, Dumas e Sainte-Beuve; in Germania lo sono Schelling, Schlegel, Hegel, dopo che Goethe lo aveva "scoperto" durante il suo viaggio in Italia nel 1787; in Russia sono ammiratori di Dante Puškin, Gogol' e più tardi Turgenev, ecc. Ma è la diffusione dei temi danteschi nella grande pittura europea già dalla fine del Settecento che dà la misura della popolarità raggiunta dall'opera sua: basti ricordare i nomi di Joshua Reynolds, William Blake, John Flaxmann, William Dyce e più tardi Dante Gabriel Rossetti in

${ }^{3}$ Cfr. Zamboni (1901). 
Inghilterra, Ingres, Carpaux, Delacroix in Francia, Asmus Jacob Carstens in Germania, Ary Scheffer in Olanda, e tanti altri. Al tempo stesso, accanto all'ammirazione per il poeta si afferma l'esigenza di un approfondimento critico della sua opera, partendo dal recupero dei testi, trasmessi con troppe incertezze e varianti di lezione dalla ricchissima tradizione manoscritta medievale e rinascimentale 4 .

Di qui, iniziative che vedono spesso studiosi stranieri all'avanguardia. Il tedesco Karl Witte, che aveva avuto modo di "scoprire" Dante durante un soggiorno romano nel 1820, dedicò la sua vita allo studio degli antichi commenti e al testo della Commedia, di cui offrì nel 1862 il primo tentativo di edizione critica moderna (seguita nel 1865 da una traduzione tedesca integrale), mentre il re Giovanni di Sassonia (König Johann von Sachsen), grande ammiratore del poeta fiorentino, sotto lo pseudonimo di Filalete ne realizzava a sua volta (tra il 1825 e il 1865 circa) una pregiata traduzione e un ampio commento che resta uno dei contributi importanti della dantologia dell'Ottocento. In Svizzera il pastore protestante Giovanni Andrea Scartazzini pubblicava tra il 1874 e il 1882 La Divina Commedia riveduta nel testo e commentata, che almeno nel commento rappresenta - dopo quello di Niccolò Tommaseo $\left(1837,1854^{2}, 1865^{3}\right)$ - il maggiore sforzo esegetico sul poema del XIX secolo. In Inghilterra Edward Moore, rettore del St. Edmund College di Oxford, in lunghi anni di studio allestiva una edizione di Tutte le Opere di Dante che, pubblicata nel 1894, s'impose subito come la nuova edizione di riferimento dell'opera dantesca (nota come Oxford Dante) ${ }^{5}$.

$\grave{E}$ in questo clima di grande fervore internazionale intorno alla figura e all'opera di Dante, di cui si sono ricordati appena gli episodi più significativi, che matura la nuova iniziativa delle "Società dantesche", mirate da un lato alla ricerca scientifica, dall' altro all'alta divulgazione, con varia prevalenza dell'uno o l'altro aspetto. Una prima associazione di "dantofili" venne costituita a Breslavia da Karl Witte dopo il suo ritorno dall'Italia, con il nome di Dante-Verein (1825): il sodalizio ebbe vita breve, ma fu poi rifondato a Dresda nel settembre 1865 come Deutsche Dante-Gesellschaft, con l'adesione di illustri studiosi, da A. Mussafia a E. Böhmer, da K. Bartsch a F. X. Wegele, a Th. Paur, presidente Witte, protettore Giovanni di Sassonia, e con il patrocinio della regina madre Elisabetta di Prussia, della regina Augusta di Prussia, della granduchessa di Weimar. Di poco posteriore è la Oxford

${ }^{4}$ Su questa fase della storia della "fortuna" di Dante v. Malato $\left(2006^{2}, 2005^{1}\right.$ : partic. $686 \mathrm{sgg}$ ). e sul concreto apporto di questo ambiente e di questa stagione di studi al progresso della ricerca dantesca, di cui ora si verrà a dire, v. Malato (2004: 43 sgg., 119 sgg. e passim).

${ }^{5}$ Per un orientamento essenziale su queste iniziative v. ancora Malato (2004: 115 sgg. e passim). 
Dante Society, a carattere inizialmente più elitario della tedesca, costituita per iniziativa di Edward Moore il 24 novembre 1876 e divenuta rapidamente il centro di raccolta di una nutrita schiera di dantisti inglesi, da H. F. Tozer a lord George Vernon, a Edm. Gardner, a Paget Toynbee (e tra gli altri il primo ministro William Ewart Gladstone, grande ammiratore e studioso di Dante $)^{6}$. Quasi simultanea, ma indipendente, fu l'iniziativa di promuovere una società dantesca americana, nata dai semi portati oltreatlantico da Lorenzo Da Ponte e altri esuli italiani, approdata nel dicembre del 1880 alla fondazione, a Cambridge (Mass.), della Dante Society of America: fondatori e animatori ne furono $\mathrm{H}$. W. Longfellow, già autore della prima traduzione americana della Commedia (1865-'67), J. S. Lowell, Ch. E. Norton, che ne furono in successione presidenti, e più tardi C. H. Grandgent, E. H. Wilkins, ecc ${ }^{7}$. Del 1888 è la fondazione, a Firenze, della Società Dantesca Italiana, che, con il patronato del re Umberto I, chiamò a raccolta i più bei nomi della cultura letteraria e della dantologia del tempo (molti non privi di peso politico), tra cui Guido Biagi, Ruggero Bonghi, Cesare Cantù, Giosue Carducci, Giuseppe Chiarini, Augusto Conti, Alessandro D'Ancona, Angelo De Gubernatis, Isidoro Del Lungo, Cesare Guasti, Guido Mazzoni, Ernesto Monaci, il promotore Carlo Negroni, Enrico Nencioni, Pio Rajna, Giuseppe Rigutini, Giovanni Tortoli, Pasquale Villari. Poco dopo, il 26 giugno 1889, per impulso di Ruggero Bonghi, il nuovo sodalizio metteva a fuoco anche il programma di lavoro della Società, a partire da una nuova edizione criticamente accertata della Divina Commedia, e s'impegnava in un'attività che nel volgere di pochi anni, con l'aggregazione di nuove forze intellettuali - Adolfo Bartoli, Giovanni Busnelli, Antonio Fiammazzo, Francesco Maggini, Salomone Morpurgo, Francesco Novati, Ernesto Giacomo Parodi, Flaminio Pellegrini, Ermenegildo Pistelli, Enrico Rostagno, Giuseppe Vandelli, cui si aggiunga un giovanissimo, poco più che ventenne, Michele Barbi, che sarebbe presto diventato l'animatore instancabile e il più attivo operatore del gruppo -, conseguì risultati importanti ${ }^{8}$.

Sono stati anni "eroici" della ricerca dantesca, italiana e straniera, che nell' arco di un secolo e mezzo o due ha esplorato tutta la documentazione su Dante e l'opera sua reperibile in tutte le biblioteche e gli archivi del mondo: un lavoro sorprendente, di cui una sorprendente testimonianza ha offerto, a metà dell'Ottocento, la Bibliografia dantesca di Paul Colomb de Bati-

${ }^{6}$ Su cui v. Toynbee $(1920,1958)$ e il vol. di Centenary Essays on Dante by Members of the Oxford Dante Society (1965). Poco aggiunge Vincent (1973).

${ }^{7}$ Notizie storiche più ampie in Gifford (1956). E v. le brevi indicazioni di Pisanti (1970) e la bibl. ivi cit.

${ }^{8} \mathrm{Sul}$ quadro qui richiamato v. Malato (2006a: 7 sgg.), da cui sono ripresi qui alcuni passaggi. 
nes, che in due tomi di circa 1200 pagine complessive (Prato, Tip. Aldina, 1845-1846), cui se ne aggiungeranno altre circa 400 di Indici e di Giunte e correzioni $(1883,1888)$, ha offerto una documentazione puntuale di tutto quanto era conservato di manoscritto e (a quell'altezza cronologica) era stato prodotto a stampa sul tema; un censimento tuttora prezioso, imprescindibile per gli studi danteschi ${ }^{9}$. Per la prima volta s'inizia l'esplorazione e si tenta il recupero degli antichi commenti - il cosiddetto "secolare commento" - alla Commedia; si promuovono nuove edizioni delle opere, sostenute da un delicato e impegnativo lavoro di restauro testuale, di selezione dell'autentico da quanto è stato abusivamente attribuito dalla tradizione, di interpretazione dei testi, di elaborazione di strumenti di consultazione e ricerca. Al tempo stesso si moltiplicano gli studi, di ampia esegesi complessiva o su singoli aspetti o passi dell'opera dantesca. Un segno della crescente attenzione popolare all'opera di Dante è offerto dalla nuova formula di lettura pubblica inaugurata a Firenze il 27 aprile 1899 e rapidamente affermatasi come Lectura Dantis, che sul modello delle antiche pubbliche letture - da Boccaccio a Benvenuto da Imola - offre una lettura e interpretazione, generalmente di un canto della Commedia, ma non solo (a volte anche gruppi di canti, o, più raramente, segmenti di canti), spesso a un livello di esegesi che senza escludere il pubblico specialistico, non respinge quello meno qualificato.

Un momento di "svolta" in questa fase storica della dantologia moderna è rappresentato dal Centenario del 1921, sesto della morte di Dante: non soltanto perché - come era già stato in passato, nel 1865, e sarà poi, nel 1965, per altri Centenari - la ricorrenza è stata occasione di studi, convegni, mostre, iniziative celebrative e di ricerca, che hanno portato un contributo notevole al progresso degli studi danteschi, ma perché in coincidenza con quella data è stata pubblicata l'edizione (nota poi come "Edizione del Centenario") di tutte Le Opere di Dante, curata da una schiera di eminenti studiosi coordinati da Michele Barbi, sotto l'egida della Società Dantesca Italiana, che per la prima volta ha offerto un'edizione "definitiva", criticamente accertata e affidabile, delle opere dantesche; con un progresso notevole rispetto al ricordato Oxford Dante di Moore e presto diventata, come per molta parte resta tuttora, l'edizione di riferimento nella lettura delle opere di Dante. Solo La Divina Commedia ha avuto una radicale (ma non sconvolgente) revisione a cura di Giorgio Petrocchi nel 1965-1966, e il De vulgari eloquentia è stato riedito da Pier Vincenzo Mengaldo nel 1968, con utilizzo dell'importante codice Berlinese, scoperto e reso fruibile dopo la pubblicazione dell'edizione del '21. Altre edizioni recenti di altre opere - dalla Monarchia di Pier Giorgio Ricci del 1965 al Convivio di Franca Ageno del 1995, dalla Vita nova di Gugliemo Gorni del 1996 alle Rime di De

${ }^{9}$ V. ora P. Colomb De Batines (2008). 
Robertis del 2002 - non hanno ottenuto quei consensi che avrebbero potuto portare a una tranquilla sostituzione dei nuovi testi a quelli dell'Edizione del Centenario, i quali restano di fatto il più sicuro punto di riferimento in una lettura scientifica, non avventurosa, delle opere di Dante ${ }^{10}$. Tanto meno ambizioni "sostitutive" possono fondatamente riconoscersi ai testi della Divina Commedia proposti da Antonio Lanza nel 1995 e da Federico Sanguineti nel 2001, che certamente non sono in grado di proporsi quale alternativa minimamente credibile al testo Petrocchi ${ }^{11}$.

Non è questo il luogo per un approfondimento delle ragioni per cui le nuove proposte testuali relative alle opere di Dante appaiono più o meno inattendibili, almeno nella loro globalità, e al di là di eventuali contributi migliorativi di singoli passi. Premeva qui mettere in evidenza come la filologia dantesca si sia particolarmente impegnata, negli ultimi due secoli, con risultati per molti versi di grande portata, sul preliminare e doveroso piano dell'autenticazione e dell'accertamento testuale delle opere di Dante, mentre parallelamente è stata condotta un'opera di scavo documentario, di approfondimento esegetico, di recupero del senso profondo del messaggio poetico di Dante, che di fatto ha aperto orizzonti nuovi alla critica dantesca e indicato direzioni di ricerca che hanno già prodotto e promettono risultati di eccezionale rilievo. Risultati - va anche questo ricordato - che la moltiplicazione degli interventi critici ha talvolta compromesso, con proposte che hanno piuttosto complicato che favorito un chiarimento del dettato dantesco (basti ricordare, per fare un solo esempio, la varietà delle proposte interpretative di un verso famoso, il "forse cui Guido vostro ebbe a disdegno" di Inf. X 63) ${ }^{12}$; ma inevitabilmente, come sempre, la verità, più o meno faticosamente, si fa strada e finisce con l'imporsi sulle indicazioni false o fuorvianti.

Siamo dunque pervenuti - sembra - a un passaggio di "svolta": di bilanci, come dicevo all'inizio, del lavoro fatto, di vaglio dei risultati conseguiti, soprattutto sul piano del restauro testuale delle opere di Dante, di valutazione degli eventuali ulteriori miglioramenti ragionevolmente prevedibili in base

${ }^{10}$ Per una ricostruzione analitica e documentata del quadro cui qui si accenna $\mathrm{v}$. Malato (2004). Partic. sull'edizione delle Rime, oltre quanto detto ivi, pp. 12-40, v. Malato (2002: 175-80); e ancora: Malato (2006a: 89-112) Beata Beatrix. Della beatitudine di Dante (e di taluni cultori di studi danteschi), partic. le pp. 89-98; ID., Di una nuova edizione commentata delle 'Rime' di Dante, ivi, a. VII 2007, 302-24. Ma v. ora del De vulgari eloquentia (2012) e della Monarchia (2013) le nuove edizioni a cura, rispettiv. di E. Fenzi e P. Chiesa \& A. Tabarroni (voll. III e IV, "Nuova Edizione commentata delle Opere di Dante (NECOD)", Roma: Salerno Editrice).

${ }^{11}$ Su tale problema v. da ultimo Malato (2007).

${ }^{12} \mathrm{Su}$ cui v. Malato (1990a). 
alla documentazione di cui si dispone; e però anche di programmazione della ricerca, la quale piuttosto che andare, come spesso è stato in passato, in direzione più o meno casuale, vede schiudersi davanti a sé nuovi ben definiti campi d'indagine, spazi di approfondimento di nuove prospettive critiche, che possono portare all'illuminazione di aspetti non soltanto rimasti in ombra, ma addirittura insospettati, del messaggio dantesco. Ed è qui, come sopra accennavo, uno degli aspetti tuttora più affascinanti dello studio di Dante, che riserva ancora, dopo settecento anni di scavi pressoché ininterrotti, di esplorazione in lungo e in largo dell'opera sua, la sorpresa di angolature nuove di ricerca, di segnali e messaggi più o meno rilevanti sapientemente nascosti nelle pieghe del dettato poetico, rimasti oscuri o comunque non còlti, o non compiutamente còlti, se non invece insospettati, nella loro effettiva valenza di "comunicazione" che l'autore intendeva trasmettere ai suoi lettori.

Su questo tema mi è capitato già di fare qualche proposta in un intervento di qualche anno fa, intitolato appunto: Nuove prospettive degli studi danteschi, pubblicato (nel 2004) come postfazione alla seconda edizione di un mio studio di alcuni anni prima (1997), dal titolo Dante e Guido Cavalcanti. Il dissidio per la 'Vita nuova'e il "disdegno" di Guido; nel quale, portando avanti uno spunto di ricerca iniziata tra il 1986 e l'89, scaturita da una lectura del canto v dell'Inferno, segnalavo come nella Commedia sia riconoscibile un'ampia presenza - accuratamente, abilmente dissimulata - di un personaggio di primissimo piano nel contesto storico e biografico di Dante, capace, per quella "presenza", di modificare in profondità la prospettiva di lettura della Commedia e non soltanto di essa: Guido Cavalcanti ${ }^{13}$. Com'è noto, Guido Cavalcanti, tradizionalmente accreditato come esponente massimo, dopo Dante, del "dolce stil novo", amico della prima giovinezza e "corrispondente" nelle prove d'esordio in rima volgare del più giovane Alighieri, dedicatario della Vita nuova, in cui si allude a lui con parole di affettuosa intesa; Guido Cavalcanti, dicevo, amico autorevole e caro, al quale più tardi, nella Commedia, Dante non esiterà a riconoscere, unico fra $\mathrm{i}$ contemporanei, un'“altezza d'ingegno" pari alla propria (quando Cavalcante, padre di Guido, gli chiederà, nel sesto cerchio dell'inferno: "Se per questo cieco / carcere vai per altezza d'ingegno, / mio figlio ov'è? e perché non è teco?": Inf. X 58-60); Guido, dunque, "presenza" dominante negli anni della giovinezza di Dante, dedicatario del libello della Vita nuova, risulta poi clamorosamente "assente" dal grande scenario del poema, dove è citato appena un paio di volte, e sempre in modo ostentatamente cursorio, quasi allusione accidentale in contesti che trattano d'altro: il ricordato "forse cui

${ }^{13}$ V. Malato $\left(2004^{2}, 1997^{1}\right)$. V. inoltre Malato $\left(2006^{2}, 2005^{1}: 50-102\right)$, Dottrina e poesia nel canto di Francesca. Lettura del canto $v$ dell' 'Inferno' (1986). 
Guido vostro ebbe a disdegno", di Inf. X 63, nel dialogo con Cavalcante, che si apre come intermezzo fortuito all'interno del più ampio colloquio con Farinata; e nel dialogo con Oderisi da Gubbio, quando il miniatore, che sta espiando il peccato di superbia nella prima cornice del purgatorio, allude ancora incidentalmente a lui, in un più articolato discorso sulla "vana gloria de l'umane posse" (Purg. XI 91): "Così ha tolto l'uno a l'altro Guido / la gloria de la lingua" (ivi, 97-98).

Il dato non è sfuggito, com'è naturale, ai commentatori antichi e ai moderni, che vi hanno variamente almanaccato sopra. Alcuni, assumendo come elemento di giudizio anche un famoso sonetto noto come la "rimenata" o "paternale" di Guido a Dante, I'vegno 'l giorno a te 'nfinite volte (Dante, Rime, XXIX; Guido, Rime, XLI), in cui Guido si rivolge all'amico con espressioni apparentemente molto severe ("tròvote, pensar troppo vilmente", "la vil tua vita", "l'anima invilita": vv. 2, 9, 14), hanno ipotizzato anche uno screzio o una rottura tra $\mathrm{i}$ due, indeterminata nelle ragioni e nei tempi, cui farebbe tuttavia qualche difficoltà l'allusione a Guido, nei due luoghi ricordati della Commedia, in termini in apparenza laudativi: nel primo caso con riconoscimento della sua "altezza d'ingegno", nel secondo con l'attribuzione a lui della palma della vittoria nella competizione con "l'altro Guido" per "la gloria de la lingua". Altri hanno negato una crisi nei loro rapporti, immaginando che fino alla fine della vita di Guido, "tra lui e Dante il colloquio sia pieno, 'corale" (Marti); ma resta inspiegata la marginalissima presenza del primo nel poema. Altri ancora hanno invece ritenuto che una frattura ci sia stata, seguita da una "chiusura" definitiva di Dante verso Guido, perciò emarginato nella Commedia: si è ritenuto che il richiamo a "l'uno" e "l'altro Guido" alluda non a Guinizzelli e Cavalcanti, bensì a Guittone e Guinizzelli, per cui si produrrebbe "nella memoria e nell'opera dantesca una linea di sbarramento [all'altezza del canto X dell'Inferno], al di là della quale il nome di Cavalcanti non si trova più" (G. Di Pino). Proposta in verità tanto più sorprendente, in quanto in evidente contrasto con tutt'altro quadro che anni prima aveva scorto l'occhio acuto di Gianfranco Contini (1966, 1970: 433), il quale, rilevando come "l'ombra e il pensiero di Cavalcanti lo accompagnano", Dante, "fino al termine d'una carriera" poetica che si conclude proprio con la Commedia, osserva che in questa "la presenza di Cavalcanti aleggia in modo tanto più inquietante quanto più indiretto: inquietante per i posteri, non per lo scrittore, i cui silenzî, le cui reticenze, le cui oscurità e ambiguità sono ferree quanto tutto il resto" 14 . Ben altro che la pretesa "chiusura" alla prima citazione nel canto X dell'Inferno.

${ }^{14}$ Una ricostruzione di tutto il quadro in Malato (2006c). Sulla sostituzione del binomio Guittone-Guinizzelli a Guinizzelli-Cavalcanti nel discorso di Bonagiunta, v. Malato (1990b). 
In ogni caso, 1'approccio tradizionale lasciava Guido nella sua apparente marginalità nel quadro generale della Commedia, più o meno circoscritta e più o meno inspiegata, sia che si ipotizzasse la rottura fra Dante e il "primo amico" della Vita nuova, sia che si immaginasse una inalterata amicizia e un perdurante "corale" colloquio tra i due, fino alla fine della vita. La prospettiva è cambiata quando, a partire dalla fine degli anni ' 80 , e a conferma della penetrante intuizione di Contini, si sono trovate cospicue tracce di Guido Cavalcanti in luoghi della Commedia dove non erano mai state neanche sospettate, e questa scoperta ha lasciato intravedere percorsi di ricerca del tutto inesplorati. Non è possibile riassumere qui il frutto di oltre vent'anni di studi, che hanno coinvolto ampi settori della dantologia internazionale e portato alla piena - ancorché non pacifica - acquisizione di dati storici e critici che proiettano una luce nuova sull'opera maggiore di Dante e sulla figura e la vicenda biografica del suo autore ${ }^{15}$. Basti ricordare che la riconosciuta diffusa "presenza" di Guido Cavalcanti in passaggi che sono snodi cruciali della Commedia, e il fatto stesso che essa sia caratterizzata dai silenzî, le reticenze, le oscurità e ambiguità denunciati da Contini, aprono una problematica inedita su tutta la costruzione dantesca, che risulta strettamente collegata al "primo amico" della giovinezza, dedicatario della Vita nuova, e portatrice di un messaggio quanto meno più denso e pregnante di quello messo a fuoco nei quasi settecento anni di esegesi precedente.

In realtà la ricerca più recente ha evidenziato come il rapporto dialogico fra Dante e Guido non si esaurisca nello scambio di pochi sonetti attestato dai manoscritti e nella dedica della Vita nuova, da parte del primo, al più anziano e autorevole amico, che non ha poi dato segno di gradimento. Si è visto come Donna me prega, per ch 'eo voglio dire, la canzone di Guido per cui egli si è guadagnato nei secoli la fama di grande poeta-filosofo, teorico d'amore, espone una dottrina dell'amore che è esattamente agli antipodi della linea di pensiero di Dante sottesa alla costruzione della Vita nuova: laddove per Guido l'amore è passione smodata, incontenibile, travolgente, tanto che "Di sua potenza segue spesso morte" (v. 35), cioè è causa di morte, morale e intellettuale, per Dante esso è "nobilissima vertù" ( $V . n$. IX 3), forza vitale, beatifica, strumento di edificazione e di elevazione a Dio, dunque causa di salvezza nella vita eterna. La divergenza è tale, che si è posto il quesito di come Dante abbia potuto dedicare il suo "libello" a Guido, nei toni "ammiccanti" che caratterizzano quella dedica, se Guido avesse già manifestato - come tradizionalmente si riteneva - tali suoi convincimenti. Si è potuto invece stabilire che non solo Donna me prega non era stata ancora scritta all'atto della dedica della Vita nuova - databile, com'è noto,

${ }^{15}$ Una rassegna dei consensi e dissensi a quella proposta, a quella data, in Malato (2007). 
intorno al 1292-93, al più tardi '94 -, ma costituisca la replica di Guido a quella dedica: di netto dissenso, contestazione puntuale delle enunciazioni teoriche fondamentali del "libello", rifiuto delle posizioni di Dante, mai nominato ma ben riconoscibile come destinatario di quella contestazione. Altri testi, fra i quali in primo luogo il ricordato sonetto di Guido I'vegno ' $l$ giorno a te 'nfinite volte, sono coinvolti in questo contrasto, che non conosce interventi di Dante. Ma non per questo si deve supporre che Dante abbia rinunciato a opporre le sue ragioni, lasciando a Guido l'ultima parola. Il rapporto si sarà certo incrinato; ma troppo forte era stato l'antico sodalizio, il legame d'amicizia, forse d'affetto, tra i due, perché la divaricazione ideologica successivamente esplosa in forme laceranti potesse anche portare a una divaricazione intellettuale, a una definitiva reciproca cancellazione di ciascuno dall'orizzonte dell'altro.

La morte di Cavalcanti, intervenuta nell'agosto del 1300, forse due o tre, al massimo quattro o cinque anni dopo la composizione di Donna me prega, può aver impedito nuove occasioni di confronto pubblico. Ma non è certo caduto il "debito" di Dante verso Guido, l'impegno suo di dare una "risposta" alle contestazioni di Guido, di cui il progresso della ricerca ha riconosciuto le tracce in vari punti del poema, coinvolto dunque a pieno titolo in questa devastante polemica tra i due più "alti ingegni" dell'ambiente intellettuale toscano dell'ultimo Duecento. La polemica, chiaramente collegata al quadro storico appena accennato, affiora in modo clamoroso in una imponente (ancorché velata) rappresentazione conflittuale di se stesso e del già "primo amico" nei canti XVI e XVIII del Purgatorio, posti in rilievo in una complessa struttura architettonica - scoperta da Charles Singleton (1978) nel 1965 -, per cui i sette canti centrali della seconda cantica, dal $\mathrm{XIV}$ al XX, sono congegnati in modo tale da costituire una sequenza chiastica incardinata sui numeri 3, 7 e 10 . Al centro di questa costruzione, che è anche il centro del poema, nel canto XVII, Dante affronta sul piano teorico il tema dell'amore, che è il tema cardine della Commedia e quello su cui si è consumata la frattura con Guido. Nei due canti contigui, XVI e XVIII, in posizione speculare, Dante rappresenta sé stesso e Guido in situazione antagonistica: sé accecato dal fumo nella cornice degli iracondi che si tira fuori dall'impasse e consegue la salvezza affidandosi a Virgilio, cioè alla guida della sua ragione ("Sì come cieco va dietro a sua guida / per non smarrirsi e per non dar di cozzo [...]": Purg. XVI 10-11); Guido a sua volta come cieco, inconsapevole di esserlo, che facendosi guida di altri ciechi - nella elaborazione teorica di Donna me prega - finisce con loro nella fossa, cioè nel peccato e nella perdizione, secondo il precetto evangelico di Matteo ("fieti manifesto / l'error de' chiechi che si fanno duci. [...]"': Purg. XVIII 17-18). È la "risposta" di Dante alla contestazione di Guido: che non 
è nominato, in quei passaggi cruciali della Commedia, come Dante non era stato nominato nella canzone contestativa della Vita nuova, ma non mancano segnali precisi, illuminanti le intenzioni allusive dei due autori. Non è possibile qui entrare nei dettagli. Ma la scoperta di questo serrato e insospettato contraddittorio nelle pieghe profonde della Commedia apre evidentemente prospettive nuove e intriganti alla esegesi dell'opera dantesca ${ }^{16}$.

Su quell'elemento strutturale, collocato certo non a caso con straordinaria evidenza, e tuttavia perfettamente mimetizzato, esattamente al centro dell'intero edificio della Commedia, poggia tutta una serie di diramazioni che ampliano e articolano il confronto dialettico tra il poeta che, smarrito nella selva oscura della irrazionalità, trova la via della salvezza recuperando la voce e la guida della ragione, e il già "primo amico", diventato irriducibile contestatore, fermo su una posizione negativa che non dà speranza all'uomo. Egli è morto da dieci o dodici anni quando Dante scrive quelle pagine, ma resta suo "interlocutore" in un dialogo virtuale ininterrotto: forse, si può anche immaginare, dall'aldilà, certo nell'attenzione dei lettori, contemporanei e posteri, che sono vivi e hanno letto le riserve di Guido e possono leggere la replica di Dante: che a quella speranza non rinuncia e vuole che non vi rinunci l'umanità, di cui egli si erge a simbolo e alla quale narra la sua esperienza salvifica perché ne sia fatta partecipe.

Alla demolizione dei capisaldi della teorica di Guido, operata da Virgilio nei suoi sermoni dottrinari (Purg. XVII 85-139; XVIII 19-39, 49-75), si aggiunge così, estesa a tutto il poema, un'ampia argomentazione accessoria, più o meno surrettiziamente collegata all'edificio principale: partendo dall'esibizione della vicenda di Paolo e Francesca, tipico caso esemplare - nel segno dell'exemplum, tanto caro alla letteratura omiletica medievale - di chi, avendo prestato fede a cattivi maestri, avendo seguito insegnamenti erronei (nel caso specifico, di Andrea Cappellano, ispiratore di tutta la letteratura cortese, che i peccatori di Rimini avevano assunto a modello di comportamento), si ritrova dannato all'inferno; fino alla rappresentazione, a sua volta esemplare, di un amore cristiano, puramente spirituale, quello fra Stazio e Virgilio: definito come "Amore / acceso di virtù", nobilitante, edificante, salvifico, che "sempre altro accese, / pur che la fiamma sua paresse fore" (Purg. XXII 10-12): capace di suscitare amore, la reciprocità del sentimento amoroso, non per forza propria, non per impulso passionale, come aveva creduto Francesca ("Amor, ch'al cor gentil ratto s'apprende [...]. Amor, ch'a nullo amato amar perdona [...]": Inf. V 100, 103), ma perché "acceso" di quella "virtù" che sola può dare valore positivo all'inclinazione amorosa. Esattamente il contrario di quanto era enunciato in Donna me prega. Il tut-

${ }^{16}$ Questo quadro, già ricostruito in Malato (1989), è stato poi ulteriormente definito in Malato (2002). 
to in una costellazione di echi, richiami, riprese dai testi cavalcantiani, in chiara funzione di segnali, per il lettore, sorprendentemente sfuggiti come tali all'esegesi storica ${ }^{17}$.

$E$ in questo quadro, in cui è ribadito alla letteratura un ruolo fondamentale di orientamento dell'uomo nel percorso della vita, non manca un ulteriore probabile intervento di rettifica a carico di Guido. La cattiva letteratura, quella dei romanzi cortesi, che sulle orme del Cappellano affermavano la natura passionale, travolgente, irresistibile dell'amore, ciecamente seguita, è stata causa della rovina di Francesca e Paolo, "i peccator carnali / che la ragion sommettono al talento" (vv. 38-39); la buona letteratura, invece, è stata la via della salvezza di Stazio, che addirittura ha potuto riscattare la sua condizione di pagano attraverso un'avveduta lettura delle opere di Virgilio, al quale dichiara, incontrandolo (Purg. XXII 64-69):

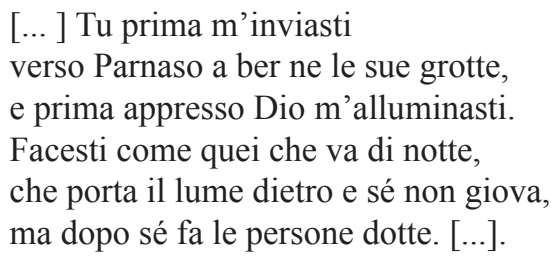

Un ruolo di altrettanto rilievo è attribuito da Dante alla nuova letteratura volgare, in particolare la lirica amorosa che segna la temperie culturale in cui hanno operato da protagonisti egli stesso e Guido, al punto che ritiene di dover riservare a quella esperienza due passaggi importanti della Commedia, nel colloquio con Bonagiunta Orbicciani (Purg. XXIV) e nell'altro con Guido Guinizzelli (Purg. XXVI), designato come l'iniziatore di quello che, con definizione dantesca, appunto, si usa definire il «dolce stil novo» (Purg. XXIV 57). Del "nuovo stile" si è ritenuto da sempre esponente massimo, a parte Dante, Guido Cavalcanti: al quale verrebbe riconosciuto il primato su Guido Guinizzelli, si è già ricordato, nella "gloria de la lingua" - benché superato, "forse", da altri, magari Dante stesso, venuto dopo di lui -, ma è negato, nel De vulgari eloquentia, né ce n'è traccia nella Commedia, il riconoscimento di cantor amoris, poeta d'amore, che è titolo onorifico esclusivo di Cino da Pistoia (D. v. e. II 2 8). Una più approfondita esplorazione dei testi, soprattutto di Cino e Guido e Dante, dei quali è possibile cogliere qualche sfumata eco nella Commedia, lascia intravedere un nuovo campo di tensione fra Dante e il già "primo amico", con coinvolgimento di Cino, per cui la divaricazione ideologica sopra ricostruita si estende al piano stilistico nella pratica poetica, per altro non distinta, o almeno non sganciata

${ }^{17}$ Un'ampia ricognizione di tali "segnali” è nella bibliogr. cit.: Amor cortese e amor cristiano, Dante e Guido, Nuove prospettive, Cavalcanti nella 'Commedia', ecc. 
dalla prima. A Dante che in Donne ch'avete intelletto d'amore, la canzone "manifesto" delle "nove rime" (Purg. XXIV 50) nella Vita nuova (V.n. XIX 4-14; Rime, XIV), ha esaltato la 'dolcezza' del linguaggio d'amore ("Amor sì dolce mi si fa sentire": v. 6) e affermato la necessità di parlare di lui in termini piani e 'leggeri' ("E io non vo' parlar sì altamente, $[\ldots] / \mathrm{ma}[\ldots]$ / a respetto di lei [Beatrice] leggeramente": vv. 9, 11, 12), che è la leujairia dei provenzali, requisito connotativo delle nuove "rime d'amor [...] dolci e leggiadre" (Purg. XXVI 99); a Dante, dicevo, che marcia decisamente in una direzione di nobilitazione del sentimento d'amore, viatico di salvezza, e di semplificazione del linguaggio lirico amoroso, dichiarata e consacrata negli incontri con Bonagiunta e con Guinizzelli, Cavalcante oppone da un lato, si è visto, la definizione 'feroce', 'punitiva', dell'amore, che "Di sua potenza segue spesso morte", dall'altro una complessità formale del discorso amoroso senza precedenti: che trova in Donna me prega una esasperazione del requisito della sottiglianza, già contestato a Cavalcanti da Guido Orlandi (nel sonetto Per troppa sottiglianza il fil si rompe: va), tale da porre quella canzone inesorabilmente fuori dal novero delle "nove rime" di cui si celebrano le lodi fra la sesta e la settima cornice del purgatorio. E si vede allora come il profilo di Cavalcanti, pur non nominato, si staglia con forte rilievo negativo nello scenario rappresentato: elogiato poco sopra per aver superato Guinizzelli "ne la gloria de la lingua", ridimensionato dall'annuncio che "forse è nato / chi l'uno e l'altro caccerà del nido" (Purg. XI 98-99), egli ritorna come "presenza"-"assenza" sulla ribalta del "dolce stil novo", di cui viene definito il profilo in termini che escludono senza possibilità di dubbio la magna canzone cui è soprattutto legata la sua figura di rimatore (e di teorico) d'amore ${ }^{18}$.

Sono soltanto alcuni spunti di uno spazio di ricerca che è ancora da definire ed esplorare in tutta la sua estensione, e promette di essere produttivo di frutti di straordinario sapore. Il recupero della figura di Guido Cavalcanti in posizione dominante in snodi cruciali della Commedia, la cura con cui essa - in attuazione di un disegno chiaramente predefinito - è stata mimetizzata da Dante in ogni parte, salvo i due luoghi di citazione esplicita, dove ancora sembra volergli dare un rilievo appena marginale, ed è invece, si è detto, di grande spessore; e ancora la frequenza degli echi, delle riprese, delle suggestioni, in molti casi certo preterintenzionali, di Cavalcanti nella Commedia, che hanno indotto Contini ad affermare che "l'elogio in fatto di Dante a Cavalcanti non cessò mai", e addirittura che "Cavalcanti aveva salato il sangue a Dante" (Contini 1970: 441, 445), sono dati che aprono prospettive di ricerca nuove e inedite, capaci di modificare in profondità il profilo della Commedia tradizionalmente acquisito.

${ }^{18}$ V. ora Malato (2006b). 
Esaurita la fase "pionieristica" della dantologia moderna, la nuova dantologia, che ha ormai tesaurizzato risultati importanti sul piano dell'indagine testuale e della documentazione storica, dovrà non solo prendere atto degli orizzonti nuovi che si schiudono alla ricerca, ma dovrà convincersi della necessità di una correzione di metodo nella conduzione della ricerca stessa. La moltiplicazione degli studi ha provocato, come si accennava all'inizio, frequenti "ingorghi" nella letteratura critica, causa non solo di difficoltà nell'aggiornamento scientifico degli studiosi, ma spesso anche di digressioni fuorvianti. Settori della dantologia chiusi in se stessi e autoreferenziali, attenti alla "novità" clamorosa molto più che all'effettiva conquista della verità, non hanno esitato a formulare proposte talvolta "originali" non meno che prive di fondamento, costruzioni fantasiose, perfino bizzarre, indotte spesso solo dalla suggestione più o meno estrosa dei proponenti, riprese e rilanciate da altri, con il risultato di portare, piuttosto che un contributo, difficoltà oggettive al progresso della conoscenza. Basti ricordare, per fare un solo esempio, la deviazione dai risultati di Contini relativi ai rapporti fra Cavalcanti e Dante, del 1965, e la riproposta, ancora trent'anni dopo, di ipotesi tanto ingegnose quanto improbabili (per non dire inverosimili). "Lo studio di Dante - avvertiva Michele Barbi (1935: 7) circa tre quarti di secolo fa - è davvero una cosa seria, che chiede come pochi altri studi vasta preparazione e lunghe meditazioni". Ciò che palesemente non è da tutti. Salvo, sempre, il diritto di tutti di esercitarsi in libere letture dei testi danteschi, pur utili alla più ampia frequentazione dell'opera di Dante, bisognerà che la critica dantesca "seria", in senso barbiano, si attrezzi con gli strumenti della filologia, l'unica che possa consentire di estrarre dai testi ciò che Dante ai testi ha affidato. Previo accertamento - com'è ovvio -, per evitare mistificazioni, di quella che possa ritenersi la più probabile autentica lezione d'autore. E la sfida che, sulle soglie del XXI secolo, alla vigilia del settimo Centenario della morte di Dante, la nuova dantologia ha davanti a sé. È certo che - pur non senza prevedibili ingorghi, contrasti, tentativi di depistaggi - sarà utilmente raccolta, e da essa trarrà alimento un rinnovato interesse del pubblico, del più vasto pubblico, per la figura $\mathrm{e}$ l'opera del nostro Poeta.

Intanto - per tornare alla domanda di partenza: perché si continua a leggere Dante oggi -, prendiamo atto della perdurante e inalterata fidelitas di questo pubblico al suo Poeta. Che sarà in parte - forse in parte - alimentata dalla problematica critica cui si è accennato, ma non solo. In verità è difficile dire cosa alimenti questa fama tenace e catturante, che non conosce cedimenti né confini, di tempo o di spazio. Certo, un ruolo importante svolge la forma del messaggio poetico dantesco, quella sua capacità di rappresentare un mondo fantastico e imprevedibile con tratti di estremo realismo, di descriverlo in quel suo linguaggio asciutto ed essenziale, sentenzioso, me- 
morizzabile, ripetibile in occasione di altre esperienze della vita di ognuno. Ma fondamento della sua intramontabile attualità è - credo - la sua vocazione a rappresentare la vita e la problematica esistenziale dell'uomo in un modo assolutamente originale, per cui negli scenari e nei personaggi della Commedia ogni lettore riconosce una proiezione e una figurazione delle sue proprie esperienze, dei suoi timori, le sue ansie, le sue aspirazioni, che sono i sentimenti propri dell'uomo, di ogni tempo e di ogni latitudine. Per altro, Dante scrive - si è ricordato - sullo scorcio del Medioevo, ormai in crisi, in un momento di passaggio, dall'età antica alla moderna, in cui l'eredità della cultura classica, filtrata dal cristianesimo, viene assunta a fondamento di una nuova cultura, che sarà quella dell'Umanesimo prima, poi dell'età che chiamiamo moderna, su cui sarà plasmata la nuova identità dell'Occidente. Accogliendo nella sua opera, con prodigiosa opera di sintesi, la somma dei fermenti, dei tormenti, delle angosce che attraversano e sconvolgono quel processo di trasformazione, Dante ha fatto della Commedia anche un documento straordinario, una testimonianza unica del processo formativo di quella nuova realtà che è la civiltà moderna. In questo senso, il poema dantesco è $-\mathrm{e}$ viene più o meno inconsciamente sentito - come un punto di riferimento culturale e identitario imprescindibile non solo dell'Italia, ma dell'intero mondo Occidentale.

\section{BIBLIOGRAFIA}

Barbi, M. (1935). Per un nuovo commento della 'Divina Commedia'. Studi Danteschi, XIX, 5-55.

Bibliografia Generale della Lingua e della Letteratura Italiana (BiGLI). (1991-). Roma: Salerno Editrice.

Centenary Essays on Dante by Members of the Oxford Dante Society. (1965). Oxford: OUP.

Contini, G. (1966). Cavalcanti in Dante. In G. Cavalcanti, Le rime (a cura di G. Contini). Verona: Officina Bedoni.

Contini, G. (1970). Cavalcanti in Dante. In G. Contini, Varianti e altra linguistica. Una raccolta di saggi (1938-1968) (pp. 433-445). Torino: Einaudi.

De Batines, P. C. (2008). Bibliografia dantesca, ossia Catalogo delle edizioni, traduzioni, codici manoscritti e comenti della 'Divina Commedia'e delle opere minori di Dante, seguito dalla serie de' biografi di lui [...] (nuova ed. anast., postfaz. a cura di S. Zamponi, Indice dei manoscritti di I. Ceccherini). Collana "Biblioteca storica dantesca", promossa dal Centro Pio Rajna, n. 3: 3 tomi. Roma: Salerno Editrice. 
Gifford, G. H. (1956). History of the Dante Society. Annual Report of the Dante Society, 3-27.

Malato, E. (1989). Amor cortese e amor cristiano da Andrea Cappellano a Dante. Studi su Dante, 571-657.

Malato, E. (1990a). Il "disdegno" di Guido. Chiosa a 'Inf.', X 63: "forse cui Guido vostro ebbe a disdegno". Studi su Dante, 425-459.

Malato, E. (1990b). Il "primato" nella "gloria de la lingua". Chiosa a 'Purg., XI, 97-98: "Così ha tolto l'uno a l'altro Guido / la gloria de la lingua". Studi su Dante, 460-92.

Malato, E. (2002). "Sì come cieco va dietro a sua guida / per non smarrirsi [...]". Lettura del canto XVI del 'Purgatorio'. Rivista di Studi Danteschi, 2, 216-57.

Malato, E. (2004a). Per una nuova edizione commentata delle Opere di Dante. Rivista di Studi Danteschi, 3-160. (Pubblicato anche in vol., Roma: Salerno Editrice, 2004).

Malato, E. (2004b). Nuove prospettive degli studi danteschi. Filologia e Critica, XXIX, 3-65.

Malato, E. (20042,1997'). Dante e Guido Cavalcanti. Il dissidio per la 'Vita nuova'e il "disdegno" di Guido (II ed. Postfazione, Nuove prospettive degli studi danteschi). Roma: Salerno Editrice.

Malato, E. $\left(2006^{2}, 2005^{1}\right)$. Il mito di Dante dal Tre al Novecento (19992001). In E. Malato, Studi su Dante. "Lecturae Dantis", chiose e altre note dantesche (pp. 658-692). Cittadella: Bertoncello Artigrafiche.

Malato, E. (2006a). In difesa della Società Dantesca Italiana. Roma: Salerno Editrice.

Malato, E. (2006b). Ancora sul "disdegno" di Guido (coinvolgendo Cino), e sul "dolce stil novo", Rivista di Studi Danteschi, I, 116-141.

Malato, E. (2006c). Cavalcanti nella 'Commedia'. Il "dialogo" ininterrotto fra Dante e Guido. Rivista di Studi Danteschi, II, 217-240.

Malato, E. (2007). Rec. a "Nuove prospettive sulla tradizione della 'Commedia'. Una guida filologico-linguistica al poema dantesco" (a cura di P. Trovato). Rivista di Studi Danteschi, 384-405.

Pisanti, T. (1970). Dante Society of America. Enciclopedia Dantesca, vol. II (p. 308). Roma: Istituto della Enciclopedia Italiana.

Singleton, C. S. (1978). Il numero del poeta al centro. In C. S. Singleton, La poesia della Divina Commedia (pp. 451-462), trad. it. Bologna: Il Mulino.

Toynbee, P. (1920). The Oxford Dante Society. Oxford: OUP.

Toynbee, P. (1958). The Oxford Dante Society after the First World War. English Miscellany, IX, 197-213. 
Vincent, E. R. (1973). Oxford Dante Society. Enciclopedia Dantesca, vol. $I V$ (pp. 238-239). Roma: Istituto della Enciclopedia Italiana.

Zamboni, M. (1901). La critica dantesca a Verona nella seconda metà del secolo XVIII. "Collezione di opuscoli danteschi inediti o rari” diretta da G. L. Passerini, vol. LXIII. Città di Castello: Lapi.

\section{READING DANTE IN THE 21ST CENTURY: THE PAST AND THE FUTURE OF DANTEAN STUDIES}

\section{Summary}

As we celebrate the seventh centenary of the publication of the Divine Comedy, and approach 2021, the seventh centenary of Dante's death, this paper offers a retrospective look at Dantean studies and summarises the work done over the past decades, while also attempting to predict further developments in this field of enquiry and to suggest some possible future directions for the Dantean critique.

Key words: Dante, seventh centenary, Divine Comedy, Dantean critique, future developments 